This item was submitted to Loughborough's Research Repository by the author.

Items in Figshare are protected by copyright, with all rights reserved, unless otherwise indicated.

\title{
Magnetic field tunable vortex diode made of YBaCuO Josephson junction asymmetrical arrays
}

PLEASE CITE THE PUBLISHED VERSION

http://dx.doi.org/10.1063/1.4997741

PUBLISHER

AIP Publishing

VERSION

VoR (Version of Record)

LICENCE

CC BY-NC-ND 4.0

\section{REPOSITORY RECORD}

Chesca, Boris, Daniel John, Richard Pollett, Marat Gaifullin, Jonathan Cox, Christopher Mellor, and Sergey Saveliev. 2019. "Magnetic Field Tunable Vortex Diode Made of Ybacuo Josephson Junction Asymmetrical Arrays". figshare. https://hdl.handle.net/2134/26149. 


\title{
Magnetic field tunable vortex diode made of $\mathrm{YBa}_{2} \mathrm{Cu}_{3} \mathrm{O}_{7-\delta}$ Josephson junction asymmetrical arrays
}

\author{
Boris Chesca, ${ }^{1}$ Daniel John, ${ }^{1}$ Richard Pollett, ${ }^{1}$ Marat Gaifullin, ${ }^{1}$ Jonathan Cox, ${ }^{1}$ \\ Christopher J. Mellor, ${ }^{2}$ and Sergey Savel'ev ${ }^{1}$ \\ ${ }^{1}$ Department of Physics, Loughborough University, Loughborough LE11 3TU, United Kingdom \\ ${ }^{2}$ School of Physics and Astronomy, University of Nottingham, Nottingham NG7 2RD, United Kingdom
}

(Received 16 May 2017; accepted 26 July 2017; published online 8 August 2017)

\begin{abstract}
Several Josephson ratchets designed as asymmetrically structured parallel-series arrays of Josephson junctions made of $\mathrm{YBa}_{2} \mathrm{Cu}_{3} \mathrm{O}_{7-\delta}$ have been fabricated. From the current-voltage characteristics measured for various values of applied magnetic field, $B$, in the temperature range of $10-89 \mathrm{~K}$, we demonstrate that the devices work as magnetic field-tunable highly reversible vortex diodes. Thus, at $89 \mathrm{~K}$, the ratchet efficiency $\eta$ could be reversed from $+60 \%$ to $-60 \%$ with a change in $B$ as small as $3 \mu \mathrm{T}$. By decreasing the operation temperature, $\eta$ improves up to $-95 \%$ at $10 \mathrm{~K}$ while the dynamics in the $B$-tunability degrades. The ratchet designs we propose here can be used to control unidirectional vortex flow vortices in superconducting devices as well as building integrated nano-magnetic sensors. Numerical simulations qualitatively confirm our experimental findings and also provide insight into the related and more general problem of the control of the transport of nano/quantum objects in thin films. Published by AIP Publishing.

[http://dx.doi.org/10.1063/1.4997741]
\end{abstract}

In the fast developing field of nanoscience, the question as to how to control nanoparticle transport on the nanoscale is very important. ${ }^{1-5}$ The role of particles drifting in nanodevices can be played not only by tiny bits of materials (say metallic or ferromagnetic spheres) but also by different excitations (quasi-particles), including those of electromagnetic, electronic, and acoustic origin. For example, an applied magnetic field $B$ penetrates a superconductor as an ensemble of magnetic flux quanta $\Phi_{0}$ known as vortices ${ }^{6}$ whose dynamics can be controlled by applied alternating, direct, or even fluctuating electrical currents. Controlling the motion of vortices in superconducting meso- and nanostructures (which are often called fluxtronic devices) has several crucial technological applications. These include the removal of noisy vortices from sensitive parts of superconducting electronics (without the inconvenience of warming-up the devices above the superconducting transition temperature) or creating a specific micro-magnetic profile in mesoscopic superconductors. ${ }^{7}$ Purpose-built devices that show a preferential direction of vortex motion are called vortex ratchet devices. Different types of vortex ratchet devices employing meso/nano-scopic superconductors with asymmetric pinning sites have been proposed (see, for example, Refs. $7-12$ ) and realized. ${ }^{13-19}$ It is important to stress that a significant feature of the fluxtronic devices is their great tunability as compared to their electronic counterparts, for instance, the vortex diode fabricated and tested in Ref. 13 allows the direction of rectification to be reversed simply by tuning the applied B. Another type of fluxtronic device based on utilizing the temporal asymmetry of applied drives to guide magnetic flux quanta ${ }^{20,21}$ has also been successfully implemented ${ }^{22-24}$ for both Abrikosov vortices (formed in bulk superconducting materials) and Josephson vortices (trapped in a Josephson junction- $J J$ - formed in a proximity region between two superconductors). Josephson ratchets based on asymmetric current bias configurations have also been fabricated. ${ }^{25-27}$ Josephson vortices are very mobile objects and can play a crucial role in both classical and quantum ratchets (see, for example, Refs. 28-30) where current reversal effects can be observed at the transition from quantum to classical regimes. ${ }^{28,31}$ Another interesting type of ratchet is based on asymmetric $J J$-arrays ${ }^{15,32}$ or asymmetric superconducting quantum interferometer devices (SQUIDs). ${ }^{33-35}$ Such ratchets are based on a device's structural/fabrication asymmetry and have the advantage of being more robust against unwanted environmental electromagnetic, electrical, or magnetic interference than other types of ratchets that have been fabricated so far. Indeed, in ratchets based on asymmetric current bias configurations, the environmental noise (of electrical/ magnetic nature) can interfere destructively with the current bias and decrease its efficiency. Ratchets based on a device's structural/fabrication asymmetry have been recently fabricated and they showed remarkable features such as a record current amplification at temperatures above $77 \mathrm{~K},{ }^{36}$ a dual flux-to-voltage response, ${ }^{37}$ and an ability to amplify the self-induced electromagnetic radiation. ${ }^{38}$ However, due to their complexity (the design consisted of a parallel-array of $22 \times 20 J J s$ connected by variable areas of SQUID-like holes), such devices have not been simulated numerically, and consequently, the physics behind their behaviour could not be fully understood. To achieve a better understanding of such ratchets based on asymmetric $J J$-structures, in this paper we report the fabrication, measurement, and numerical simulation of three less complex designs.

The $J J$-arrays were fabricated by depositing high quality epitaxial, $100 \mathrm{~nm}$ thick $c$-axis oriented $\mathrm{YBa}_{2} \mathrm{Cu}_{3} \mathrm{O}_{7-\delta}(\mathrm{YBCO})$ films on $10 \times 10 \mathrm{~mm}^{2}, 24^{\circ}$ symmetric [001] tilt $\mathrm{SrTiO}_{3}$ bicrystals by pulsed laser deposition. A $200 \mathrm{~nm}$ thick Au layer was deposited in situ on top of the YBCO film to facilitate fabrication of high quality electrical contacts for electric transport measurements. The films, with a critical temperature of $T_{c}$ of 


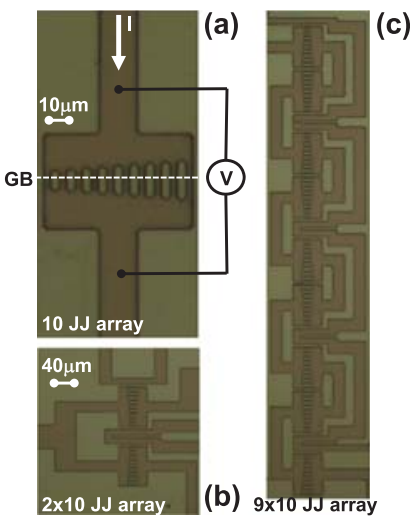

FIG. 1. Optical micrographs showing three devices consisting of (a) a single parallel array of 10 Josephson junctions and (b) 2 and (c) 9 such identical parallel arrays connected in series. The bicrystal grain boundary (GB) is shown by the dotted line in (a). The Josephson junctions are formed across the GB and can be seen as bridges, grey in colour, crossing the GB. Within each parallel array, the rectangular holes separating the junctions have an identical width of $3 \mu \mathrm{m}$ and increasing lengths from 8 to $16 \mu \mathrm{m}$.

$92 \mathrm{~K}$, were subsequently patterned by optical lithography and etched by an Ar ion beam to form either a single asymmetric parallel array of $10 J J_{S}$ [see Fig. 1(a)], 2 [see Fig. 1(b)], or 9 identical such arrays connected in series [Fig. 1(c)]. Within each such parallel array, all $10 J J_{s}$ are $3 \mu \mathrm{m}$ wide. The junctions are separated by superconducting loops of identical width of $3 \mu \mathrm{m}$ but variable length. The loops' length increases linearly from $8 \mu \mathrm{m}$ to $16 \mu \mathrm{m}$ in steps of $1 \mu \mathrm{m}$. Since the individual SQUID inductances, $L_{n}$, are proportional to the SQUID loop perimeter $(1 \mu \mathrm{m}$ corresponds to approximately $1 \mathrm{pH})$ $\beta_{L n}=2 \pi L_{n} I_{c} / \Phi_{0}$ also increases monotonically by $58 \%$ within the $10 J J_{S}$-array, with $\mathrm{n}=1,2, \ldots, 9$ where $I_{c}$ is the $J J_{S}$ critical current. One can therefore define an average value $\beta=\left\langle\beta_{L n}\right\rangle$ for the array. $\beta$ can be estimated from both the modulation of $I_{c}$ with $B$ or direct calculations. At $89 \mathrm{~K}$, we estimated that $\beta_{L n}$ varies from 0.27 to 0.54 within the array, while at $10 \mathrm{~K}$ it varies from 50 to 100 . The bias current $I$ is applied symmetrically via the central top and bottom electrodes and $V$ is measured across the array. $B$ is applied perpendicular to the planar
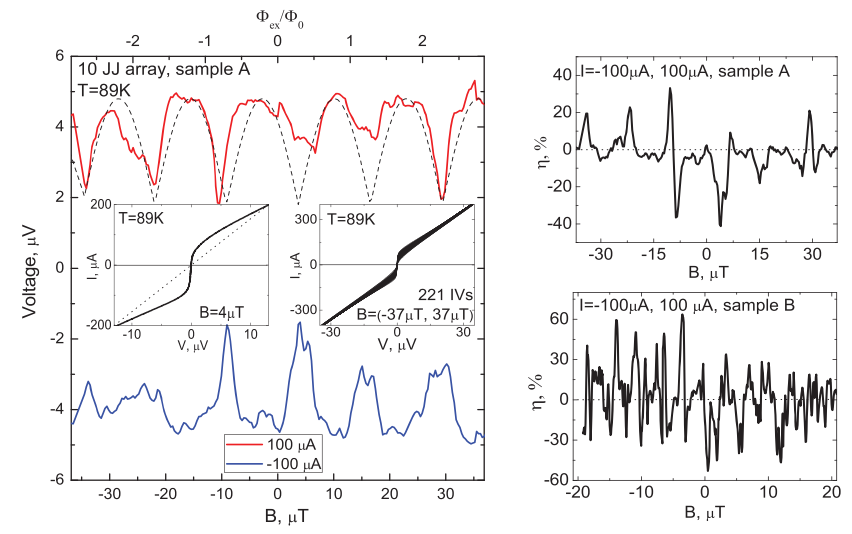

FIG. 2. Electrical transport properties of parallel 10JJ-arrays at $89 \mathrm{~K}$ (samples A and B). On the left-hand side, $V(B)$ is shown for two different values of the bias current, $I$. The $V(B)$ characteristics were obtained from families of $I V C$ s taken at multiple $B$ field values (shown in the insets: a single $I V C$ and $221 I V C s$ ). With the dashed line in (a), the periodical theoretical curve of $V(B)$ is shown calculated using the approach developed in. ${ }^{39}$ From $V(B)$, the ratchet efficiency $\eta(\%)$ has been calculated (right-hand side figures).
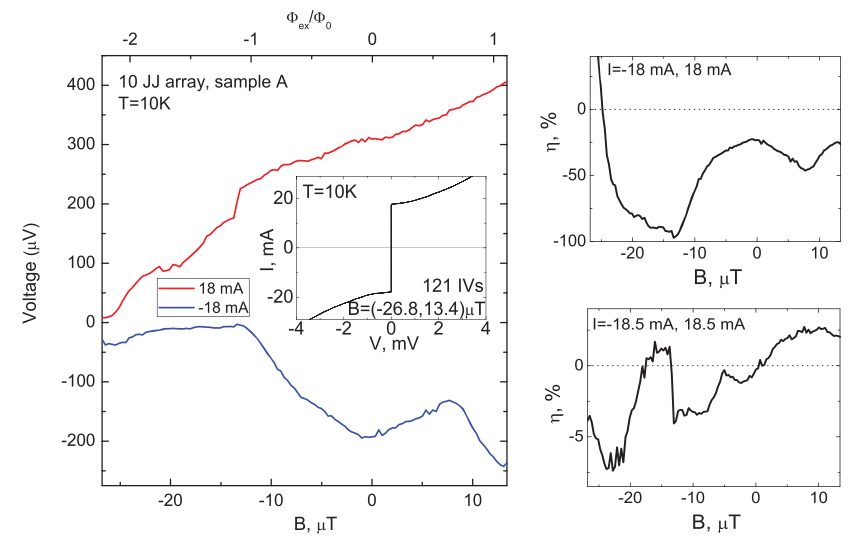

FIG. 3. Electrical transport properties of a parallel 10JJ-array at $10 \mathrm{~K}$ (sample A). On the left-hand side, $V(B)$ is shown for two different values of the bias current, $I$. The $V(B)$ characteristics were obtained from families of $I V C s$ taken at multiple $B$ field values (shown in the inset: $121 \mathrm{IVs}$ ). From $V(B)$, the ratchet efficiency $\eta(\%)$ has been calculated (right-hand side figures).

array's structure via a control current $I_{c t r l}$ through an inductively coupled coil. Consequently, an external magnetic flux, $\Phi_{e x}$, is coupled into the array. We fabricated two devices for each of the three designs considered here, and for each of the designs, both devices showed a qualitatively similar behaviour. Since there is no visible hysteresis in the current-voltage characteristics $(I V C S)$ measured, the capacitance of our $J J_{S}$ can be considered to be negligible. Therefore, each $J J$-array can be modelled as an array of resistively shunted junctions connected via superconducting inductances.

Families of IVCs were measured by a 4 point-contact method at various temperatures $T$ between $10 \mathrm{~K}$ and $92 \mathrm{~K}$ and for different values of $I_{\text {ctrl }}$ in the range of $-8 \mathrm{~mA}$ to $8 \mathrm{~mA}$. $I_{\text {ctrl }}$ was changed in small steps of $15 \mu \mathrm{A}$. Such families of consecutive IVCs measured at different temperatures are plotted in the left hand side insets of Figs. 2 and 3. From such families of $I V C s$ scanned over $I_{c t r l}$ (or equivalently, $B$ or $\left.\Phi_{e x}\right), V(B)$ for both positive and negative bias currents has been constructed (see Figs. 2-4). The corresponding $I_{c}(B)$ curves look qualitatively similar to the $V(B)$ plots. At temperatures close to $T_{c}, \beta_{L n} / \pi$ is negligibly small. In this limit, $V\left(\Phi_{e x}\right)$ and $I_{c}\left(\Phi_{e x}\right)$ for a symmetrical parallel $10 \mathrm{JJ}$-array (i.e., an array with identical SQUID holes) should have a periodic pattern consisting of a series of maxima (shown by

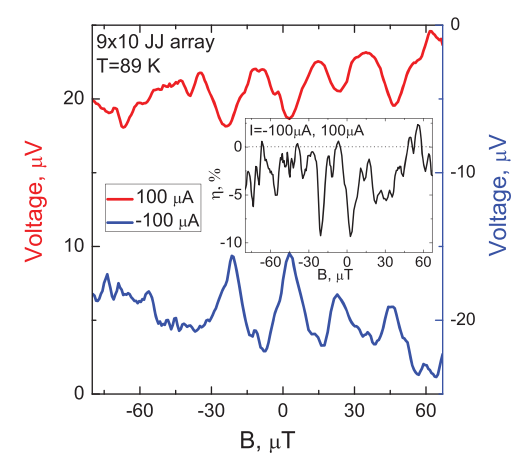

FIG. 4. Electrical transport properties at $\mathrm{T}=89 \mathrm{~K}$ of a parallel-series device consisting of nine 10JJ parallel arrays connected in series. $V(B)$ is shown for two different values of the bias current, $I$. From $V(B)$, the ratchet efficiency $\eta(\%)$ has been calculated (right-hand side inset). 
a dashed line in Fig. 2) similar to the diffraction pattern of a multiple slit optical grating. ${ }^{39}$ The flux periodicity corresponds to one additional $\Phi_{0}$ in each loop. It is remarkable that although the arrays we measured are asymmetric, qualitatively the flux periodicity expected for a symmetric design is still visible to some degree in the experiments carried out at $89 \mathrm{~K}$ (see Figs. 2 and 4). As expected, with decreasing temperature $I_{c}$ and the average $\beta_{L n}$, both increase and consequently any trace of periodicity in the $V(B)$ vanish quickly (see measurements taken at $10 \mathrm{~K}$ shown in Fig. 3). From the $V(B)$ curves, the ratchet efficiency can be calculated as: $\eta=\frac{V(I)+V(-I)}{V(I)-V(-I)} \times 100 \%$. The $\eta(B)$ dependencies for various values of $I$ are shown in Figs. 2-4.

To be able to interpret the $\eta(B)$ dependencies, one has to first understand the physics involved. When $B$ is applied perpendicularly to a $J J$-array plane, some flux quanta are trapped in the array loops. I flowing across the array produces a Lorentz force which drives the flux quanta unidirectionally, forming a lattice of vortices moving with a speed, $v$. This vortex motion produces a dc voltage $V$ across the array which can be measured. In a symmetrical array (i.e., an array with identical loop areas and junctions), the intensity of the flux flow (and consequently absolute value of $V$ ) is identical for both positive and negative $I$. As a result, as confirmed by our simulations, $\eta(B)$ is always zero, independent of the value of $B$ [see Fig. 5(a)]. In our experiments, however, for most values of $B, \eta(B)$ is very different from zero with values above $40 \%$ at all measured temperatures and reaching almost $100 \%$ at $10 \mathrm{~K}$ for particular values of $B$. This suggests that the vortex flux flow is highly preferential in one direction, i.e., the device operates as a vortex diode. Moreover, as we change $B$, $\eta(B)$ changes its sign repeatedly, suggesting that the device works as a $B$-tunable (highly reversible) Josephson vortex diode. Thus, at $89 \mathrm{~K}, \eta(B)$ could be reversed from $+60 \%$ to $-60 \%$ (sample B) and from $+40 \%$ to $-40 \%$ (sample A) with a change in $B$ as small as $3 \mu \mathrm{T}$ (see Fig. 2). The dynamics in the $B$-tunability is significantly supressed with decreasing temperature. Thus, for a single $10 \mathrm{JJ}$ parallel array, $\eta$ switches from positive to negative values 8 times within a $80 \mu \mathrm{T}$ range at $89 \mathrm{~K}$ (see Fig. 2), but it does so only once or twice within a $40 \mu \mathrm{T}$ range at $10 \mathrm{~K}$ (see Fig. 3). Such $B$-tunability can find application in electronic devices based on multiple $J J_{S}$ such as rapid single flux quantum (RSFQ), ${ }^{40}$ SQUIDs, ${ }^{41}$ or superconducting quantum interference filters $(\mathrm{SQIFs})^{42,43}$ when a tunable preferential direction of flux flow is required. In order to qualitatively understand why efficiency changes sign when $B$ is varied, it is important to understand that usually, during the device's operation, there are many $\Phi_{0}$ per array loop involved in the ratchet's dynamics. Therefore, instead of analysing the vortex motion of all the vortices, one can instead look at the dynamics of flux interstitials only, which are extra vortex quanta or one missed vortex relative to a perfect matching configuration of integer $\Phi_{0}$ per array loop at a given applied $B$. Since the extra $\Phi_{0}$ and the vortex holes (i.e., a missing vortex) have opposite preferred direction of motion (since they have opposite "magnetic charge") and the transition from an extra vortex dominated picture to a hole dominated picture occurs when magnetic flux varies by one flux quanta per smallest array loop area in the array, the efficiency
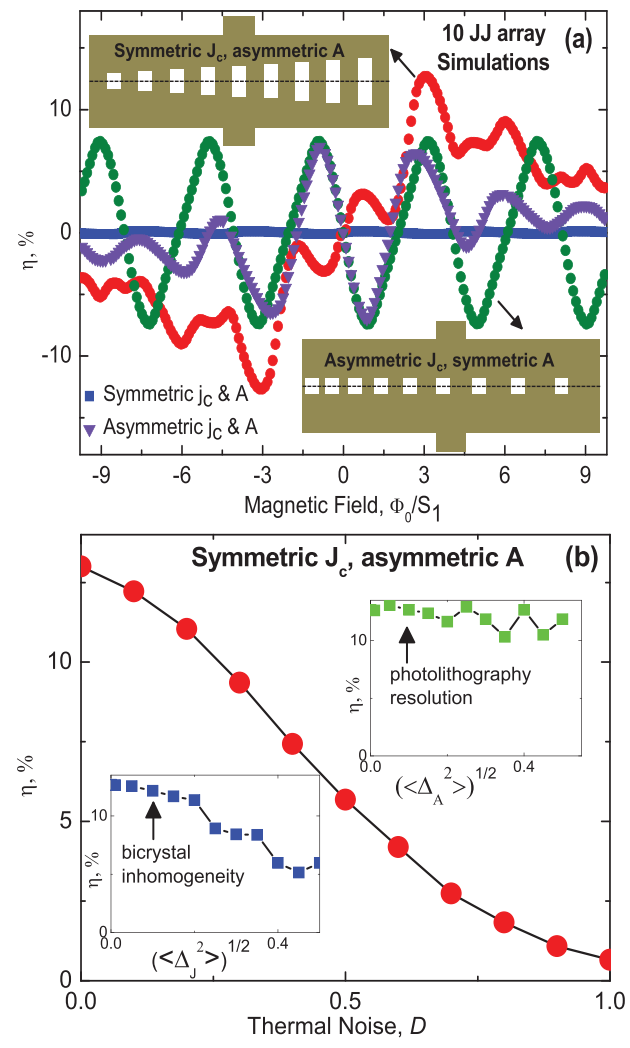

FIG. 5. (a) $\eta(\%)$ versus B for three possible ways of ratchet asymmetry implementation: symmetric $j_{c n}$ and asymmetric $A_{n}$ (top-left design and red solid circles curve); asymmetric $j_{c n}$ and symmetric $A_{n}$ (bottom-right design and green solid circle curve); asymmetric $j_{c n}$ and $A_{n}$; the case of symmetric $j_{c n}$ and $A_{n}$ when no ratchet effect is observed is shown too; (b) the effect of fluctuations on $\eta$ calculated for a ratchet with symmetric $j_{c n}$ and asymmetric $A_{n}$ and a bias current of $10 \%$ above the Josephson critical current. Three types of fluctuations are considered: thermal noise, $\mathrm{j}_{\mathrm{c}}$ fluctuations (lower inset), and fluctuations in the array loop area (upper inset).

will change sign when $B$ is varied. This is in agreement with our measurements.

Self-induced magnetic fields of the order $L_{\text {average }} I$ are generated by the carrying lines. Here, $L_{\text {average }}=30 \mathrm{pH}$ is the average array loop inductance and $I$ is the bias current, typically slightly larger than the Josephson critical current (about $100 \mu \mathrm{A}$ at $89 \mathrm{~K}$ and $18 \mathrm{~mA}$ at $10 \mathrm{~K}) . L_{\text {average }} I$ takes therefore values between $\left(6 \times 10^{-7}\right.$ and $\left.3 \times 10^{-9}\right) \quad \mu \mathrm{T}$ as we change temperature in the range of $10-89 \mathrm{~K}$. These values are negligibly small relative to a few $\mu \mathrm{T}$ required to significantly modulate $\eta(B)$ (see Figs. 2-4). This also suggests that our ratchets are quite robust against unwanted environmental electromagnetic noise which typically will induce currents in the range of $n A$. Such small currents will be insufficient to produce magnetic fields that will affect the ratchet point of operation.

At $89 \mathrm{~K}, \eta(B)$ maxima of single parallel $10 \mathrm{JJ}$-arrays [Fig. 1(a)] are significantly larger (see right-hand side graphs in Fig. 2) than a maximum of about $30 \%$ reached for a design consisting of 2 such arrays connected in series [Fig. 1(b)] and much larger than about $10 \%$ (see inset in Fig. 4) reached for a design consisting of 9 such arrays in series [Fig. 1(c)]. A similar behaviour has been observed at all temperatures in the range of $10-89 \mathrm{~K}: \eta(B)$ is the highest for parallel $10 \mathrm{JJ}$ arrays, is significantly lower for 2 serially connected 10 
$\mathrm{JJ}$-arrays, and is the smallest for the 9 serially connected 10 JJ-arrays. These observations suggest that when $\mathrm{N}$ parallelarrays are connected in series, the ratchet efficiency decreases rapidly with $\mathrm{N}$. This effect can be easily understood if considering the spread of $I_{c}$ of the individual $J J$-arrays connected. ${ }^{41}$ Indeed, since the resulting voltage of $\mathrm{N}$ parallel $10 \mathrm{JJ}$-arrays connected in series is the sum of all individual voltages for all bias currents taking values in the range (minimum $I_{c}$ to maximum $I_{c}$ ), some $10 \mathrm{JJ}$-arrays will be in the voltage state showing some ratchet efficiency (positive or negative) and some will be in the zero-voltage state showing no ratchet efficiency. Consequently, the overall effect on the entire device's ratchet efficiency will be diluted when connecting $\mathrm{N}$ parallel $10 \mathrm{JJ}$-arrays in series relative to a single $10 \mathrm{JJ}$-array.

The design asymmetry of the JJ-arrays can be implemented in three different ways using the asymmetry in the Josephson critical currents of nth junction $j_{c n}$ or/and in the array loop areas $A_{n}$ : (i) symmetric $j_{c n}$ and asymmetric $A_{n}$; (ii) asymmetric $j_{c n}$ and symmetric $A_{n}$; and (iii) asymmetric $j_{c n}$ and $A_{n}$; Simulations show [see Fig. 5(a)] that in order to maximise efficiency $\eta$, the best way to implement asymmetry is by keeping $j_{c n}$ of all junctions the same and varying $A_{n}$ (symmetric $j_{c n}$ and asymmetric $A_{n}$ ). It is for this reason that this case has been realized in practice.

The asymmetric Josephson transmission line can be described by a set of coupled ordinary differential equations ${ }^{44}$

$$
\begin{aligned}
\frac{d \varphi_{0}}{d t}= & j-j_{c 0}\left(1+\Delta_{J}\right) \sin \varphi_{0}+\alpha\left[\frac{\varphi_{1}-\varphi_{0}}{\left(1+\Delta_{A}\right) A_{\frac{1}{2}}}-h\right] \\
& +\sqrt{2 D} \xi_{0} \\
\frac{d \varphi_{n}}{d t}= & j-j_{c n}\left(1+\Delta_{J}\right) \sin \varphi_{n}+\alpha\left[\frac{\varphi_{n+1}-\varphi_{n}}{\left(1+\Delta_{A}\right) A_{n+\frac{1}{2}}}\right. \\
& \left.-\frac{\varphi_{n}-\varphi_{n-1}}{\left(1+\Delta_{\mathrm{A}}\right) A_{n-\frac{1}{2}}}\right]+\sqrt{2 D} \xi_{n} \\
\frac{d \varphi_{n_{\max }}=}{d t} & j-j_{c n_{\max }}\left(1+\Delta_{J}\right) \varphi_{n_{\max }}+\alpha\left[h-\frac{\varphi_{n_{\max }}-\varphi_{n_{\max -1}}}{\left(1+\Delta_{A}\right) A_{n_{\max }-\frac{1}{2}}}\right] \\
& +\sqrt{2 D} \xi_{n_{\max } .}
\end{aligned}
$$

In order to match the experimentally implemented design, $n$ runs from 1 to 8 in the middle equation of set (1) while $\mathrm{n}_{\max }=9$ in the last equation of the set. We denote the dimensionless applied current as $\mathrm{j}=\mathrm{I} /\left\langle I_{c}\right\rangle$, with $\left\langle I_{c}\right\rangle$ being the array's average $I_{c}, \varphi_{n}$ (with $n=0, \ldots, 9$ ) is the gauge invariant phase difference across the nth junction, dimensionless time $t$ is measured in the units of the characteristic relaxation time $\tau=\Phi_{0} / 2 \pi c R I_{\mathrm{c}}$, with $\Phi_{0}$ being the flux quantum, $c$ the speed of light, and $R$ the junction resistance (assumed the same for all junctions). The detailed consideration of two dimensional boundary conditions of the problem suggests the same $j$ in all junctions of the line. We also introduce parameter $\alpha=\tau c R /\left(4 \pi \mathrm{aS}_{1 / 2}\right)$ with the inter-junction distance $a$ and the smallest area $S_{1 / 2}$ of the array loop between junctions with $n=0$ and $n=1$. The array loop area linearly increases with the junction number and area ratio $A_{\mathrm{n}+1 / 2}$ $=S_{\mathrm{n}+1 / 2} / S_{1 / 2}=1+n / 8$, with $\mathrm{n}+1 / 2$ being the array loop between $n$th and $(n+1)$ th junctions. The dimensionless magnetic flux $h=H S_{1 / 2} / \Phi_{0}$ is measured in units of magnetic flux quantum per smallest loop area of the array. In the simulations, we also considered the effect of two types of structural fluctuations related to the $J J$-arrays: (i) critical current fluctuations from junction to junction $\Delta_{J}$, with the average $\left\langle\Delta_{J}\right\rangle=0$, i.e., $I_{c n}$ of the $n$th junction can vary randomly from the designed value (due to inhomogeneities of the bicrystal line ${ }^{41}$ ) resulting in fluctuations of $j_{c n}=I_{c n} /\left\langle I_{c}\right\rangle$; (ii) fluctuations in the array loop areas $\Delta_{A}$, with the average $\left\langle\Delta_{A}\right\rangle=0$, due to the $1 \mu \mathrm{m}$ finite resolution of photolithography used in the fabrication process. Finally, thermal fluctuations have been considered too: temporal unbiased $\delta$-correlated Gaussian white noise $\xi_{n}$ with intensity $D$, i.e., $\left\langle\xi_{n}\right\rangle=0,\left\langle\xi_{n}(0) \xi_{n}(t)\right\rangle=\delta(t)$, which can occur, for instance, due to thermal noise or temporal current fluctuations of the external circuit. In order to estimate $\eta$, we average the time derivative of the gauge invariant phase difference over time and junctions $\overline{\dot{\varphi}}(j)=\left\langle\left\langle d \varphi_{n} / d t\right\rangle_{n}\right\rangle_{t}$ and use the dimensionless ratchet efficiency, $\eta=\frac{\overline{\dot{\varphi}}(j)+\overline{\dot{\varphi}}(-j)}{\dot{\varphi}(j)-\bar{\varphi}(-j)} \times 100 \%$. The effect on $\eta$ of all these three types of fluctuations is shown in Fig. 5(b). It is important to notice that while large thermal fluctuations $(D>0.5)$ or critical current fluctuations significantly supress $\eta$, moderate fluctuations in the array loop areas $\left(\left\langle\Delta_{A}^{2}\right\rangle^{1 / 2}\right.$ in the range $\left.(0-0.5)\right)$ have an insignificant effect on $\eta$. The corresponding experimental points (indicated by vertical arrows) are shown in the insets of Fig. 5(b).

To compare the simulation results with the experimental data, we consider $\eta(B)$ at a fixed value of $I$. The red curve in Fig. 6 represents results without structural fluctuations $\left(\Delta_{J}=0\right.$ and $\Delta_{A}=0$ ) and temporal noise $D=0$. In this case, it is possible to show that any solution of Eq. (1) should be periodic as a function of $h$ with period $\Delta h=16 \pi$, which is clearly seen in the upper inset of Fig. 6 (red curve). Spatial fluctuations of $I_{c n}$ eliminate such fragile periodicity (upper inset in Fig. 6, blue curve), making it very difficult to observe experimentally (no experimental evidence has been found in our data). In order to make a qualitative comparison with the experiment results, we plot an enlarged part of the upper inset in the main panel of Fig. 6. Comparing the simulated result with, say, experimental data shown in Figs. 2-4, we conclude that the model captures the main experimental features well: the order of magnitude of the ratchet effect, the variation of $\eta$ with $B$, and the change of rectification directions (reversible rectification tuned by $B$ ). Comparing the simulated rectification with and without spatial fluctuations of the critical current, we also expect that each sample (where such fluctuations are unavoidable and uncontrollable) should have its individual fingerprint, thus requiring individual calibration for practical usage. Also, the presence of the Earth's magnetic field $B_{E}$ generates a shift in the magnetic field axis, resulting in some additional uncertainty for quantitative comparison of experimental data and simulations. The amount of flux coupled into the array due to $B_{E}$ is also difficult to estimate considering the well-known large flux focusing effects in bicrystal Josephson junctions. ${ }^{41,42}$ For these reasons, here we restrict ourselves with only a qualitative comparison between the model and the experimental data and this indicates good agreement between them.

Taking advantage of the ability to explore a large temperature range in detail within our simulations, we vary temperature 


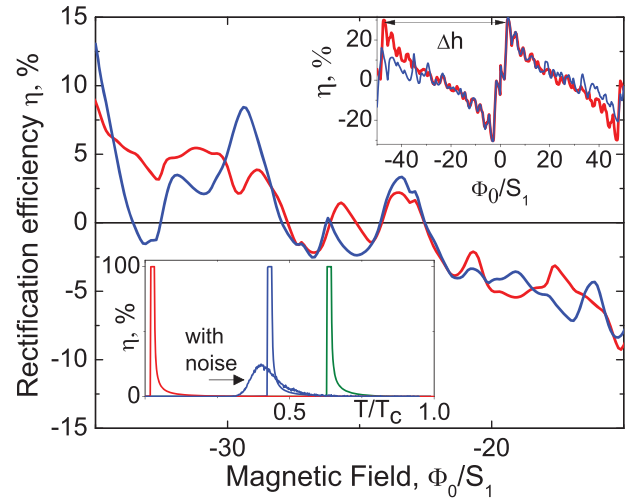

FIG. 6. Electrical transport properties simulated by using Eq. (1). The ratchet efficiency $\eta(h)=\frac{\overline{\dot{\varphi}}(j, h)+\overline{\dot{\varphi}}(-j, h)}{\dot{\bar{\varphi}}(j, h)-\overline{\bar{\varphi}}(-j, h)}$ as a function of the applied magnetic field $h$ for applied current $j=1, \alpha=0.1, D=0, j_{c n}=1$ (for all $n$ for red curve), and one realization of random $j_{c n}$, which is homogeneously distributed in the interval $[0.5,1.5]$ (for blue curve). The curves in the upper inset are the same as in the main panel but for larger magnetic field range where periodicity is clearly seen for (red) but not for the blue curve. Lower inset: the temperature dependence of the ratchet efficiency $\eta(T)=\frac{\overline{\dot{\varphi}}(j, T)+\overline{\dot{\varphi}}(-j, T)}{\dot{\dot{\varphi}}(j, T)-\overline{\dot{\varphi}}(-j, T)}$ for $h=1, \alpha=0.1$; no temporal noise is included in the curves reaching $100 \%$ efficiency (i.e., $D=0): j_{c n}=\left(1-T / T_{c}\right)$ (for all $n$ ); and the applied current $j=0.9$ (red curve), $j=0.5$ (blue curve), and $j=0.3$ (green curve). The ratchet efficiency for $h=1, \alpha=0.1, j_{c n}=\left(1-T / T_{c}\right)$, and $j=0.5$ in the presence of temporal noise $\left(\mathrm{D}=\mathrm{D}_{0} \mathrm{~T} / \mathrm{T}_{\mathrm{c}}, \mathrm{D}_{0}=0.05\right)$, shown again in blue, is drastically reduced to about $25 \%$ in accordance to typical values observed in the experiments at high temperatures.

from very low values to $T_{\mathrm{c}}$ at fixed values for $I$ and $B \cdot \eta(T)$ is shown in the lower inset of Fig. 6. In contrast to the quite unpredictable dependence of $\eta(B)$ (Fig. 6), $\eta(T)$ exhibits a clear peak (lower inset of Fig. 6) when the applied current reaches the critical current of the sample $j=j_{c n}\left(T_{s w}\right)$ (or its minimum value if $j_{c n}$ fluctuates) that occurs at a certain welldefined temperature, $T_{s w}$. This could be used to design a temperature switch which changes its state when the temperature crosses the value, $T_{s w}(h)$. Importantly, the switching temperature can be tuned by the applied $B$. This effect is robust with respect to a certain amount of white noise (lower inset of Fig. 6) which further justifies the potential of the discussed effect for temperature switching applications.

The operation of magnetically tunable Josephson ratchets designed as asymmetrically structured parallel or parallel-series $J J$ arrays made of $\mathrm{YBa}_{2} \mathrm{Cu}_{3} \mathrm{O}_{7-\delta}$ has been demonstrated in a wide temperature range of $10-89 \mathrm{~K}$. The ratchet efficiency $\eta$ could be very efficiently controlled by $B$ : at $10 \mathrm{~K}, \eta$ drops from $-95 \%$ to $0 \%$ when $B$ changes by $12 \mu \mathrm{T}$ (see Fig. 3), while at $89 \mathrm{~K}, \eta$ could be reversed from $+60 \%$ to $-60 \%$ (in sample B) or from $+40 \%$ to $-40 \%$ (sample A) with a change in $B$ as small as $3 \mu \mathrm{T}$ (see Fig. 2). Arrays connected in parallel have a significantly improved ratchet efficiency $\eta$ in comparison to parallel-series arrays. Such vortex diodes are excellent potential candidates in applications where controlling unidirectional vortex flow is essential such as SQUIDs, SQIFs, or RSFQ.

${ }^{1}$ P. Hänggi and F. Marchesoni, Rev. Mod. Phys. 81, 387 (2009).

${ }^{2}$ P. Hänggi, F. Marchesoni, and F. Nori, Ann. Phys. 14, 51 (2005).

${ }^{3}$ S. Savel'ev and F. Nori, Chaos 15, 026112 (2005).

${ }^{4}$ C. Bustamante, J. Liphardt, and F. Ritort, Phys. Today 58(7), 43 (2005).
${ }^{5}$ J. Huguet, C. V. Bizarro, N. Forns, S. B. Smith, C. Bustamante, and F. Ritort, Proc. Natl. Acad. Sci. U.S.A. 107, 15431 (2010).

${ }^{6} \mathrm{M}$. Tinkham, Introduction to Superconductivity, 2nd Revised ed. (Dover Publications, Inc., 2004)

${ }^{7}$ C. S. Lee, B. Janko, I. Derenyi, and A. L. Barabasi, Nature 400, 337 (1999).

${ }^{8}$ J. F. Wambaugh, C. Reichhardt, C. J. Olson, F. Marchesoni, and F. Nori, Phys. Rev. Lett. 83, 5106 (1999).

${ }^{9}$ C. J. Olson, C. Reichhardt, B. Janko, and F. Nori, Phys. Rev. Lett. 87, $177002(2001)$.

${ }^{10}$ S. Savel'ev, F. Marchesoni, and F. Nori, Phys. Rev. Lett. 91, 010601 (2003).

${ }^{11}$ S. Savel'ev, F. Marchesoni, and F. Nori, Phys. Rev. Lett. 92, 160602 (2004).

${ }^{12}$ S. Savel'ev, F. Marchesoni, and F. Nori, Phys. Rev. E 70, 061107 (2004).

${ }^{13}$ J. E. Villegas, S. Savel'ev, F. Nori, E. M. Gonzalez, J. V. Anguita, R. García, and J. L. Vicent, Science 302, 1188 (2003).

${ }^{14}$ J. B. Majer, J. Peguiron, M. Grifoni, M. Tusveld, and J. E. Mooij, Phys. Rev. Lett. 90, 056802 (2003).

${ }^{15}$ D. E. Shalom and H. Pastoriza, Phys. Rev. Lett. 94, 177001 (2005).

${ }^{16}$ M. Beck, E. Goldobin, M. Neuhaus, M. Siegel, R. Kleiner, and D. Koelle, Phys. Rev. Lett. 95, 090603 (2005)

${ }^{17}$ J. Van de Vondel, C. C. de Souza Silva, B. Y. Zhu, M. Morelle, and V. V. Moshchalkov, Phys. Rev. Lett. 94, 057003 (2005).

${ }^{18}$ Y. Togawa, K. Harada, T. Akashi, H. Kasai, T. Matsuda, F. Nori, A Maeda, and A. Tonomura, Phys. Rev. Lett. 95, 087002 (2005).

${ }^{19}$ C. C. de Souza Silva, J. Van de Vondel, M. Morelle, and V. V. Moshchalkov, Nature 440, 651 (2006).

${ }^{20}$ S. Savel'ev and F. Nori, Nat. Mater. 1, 179 (2002).

${ }^{21}$ S. Savel'ev, F. Marchesoni, P. Hänggi, and F. Nori, Europhys. Lett. 67, 179 (2004); Eur. Phys. J. B 40, 403 (2004).

${ }^{22}$ D. Cole, S. Bending, S. Savel'ev, A. Grigorenko, T. Tamegai, and F. Nori, Nat. Mater. 5, 305 (2006).

${ }^{23}$ S. Ooi, S. Savel'ev, M. B. Gaifullin, T. Mochiku, K. Hirata, and F. Nori, Phys. Rev. Lett. 99, 207003 (2007).

${ }^{24}$ A. V. Ustinov, C. Coqui, A. Kemp, Y. Zolotaryuk, and M. Salerno, Phys. Rev. Lett. 93, 087001 (2004).

${ }^{25}$ R. Gerdemann, T. Bauch, O. M. Froehlich, L. Alff, A. Beck, D. Koelle, and R. Gross, Appl. Phys. Lett. 67, 1010 (1995).

${ }^{26}$ J. Schuler, S. Weiss, T. Bauch, A. Marx, D. Koelle, and R. Gross, Appl. Phys. Lett. 78, 1095 (2001).

${ }^{27}$ T. Bauch, S. Weis, H. Haensel, A. Marx, D. Koelle, and R. Gross, IEEE Trans. Appl. Supercond. 7, 3605 (1997).

${ }^{28}$ A. O. Sboychakov, S. Savel'ev, A. L. Rakhmanov, and F. Nori, Phys. Rev. Lett. 104, 190602 (2010).

${ }^{29}$ S. Savel'ev, A. L. Rakhmanov, and F. Nori, Phys. Rev. Lett. 98, 077002 (2007).

${ }^{30}$ S. Butz, A. K. Feofanov, K. G. Fedorov, H. Rotzinger, A. U. Thomann, B. Mackrodt, R. Dolata, V. B. Geshkenbein, G. Blatter, and A. V. Ustinov, Phys. Rev. Lett. 113, 247005 (2014).

${ }^{31}$ P. Reimann, M. Grifoni, and P. Hänggi, Phys. Rev. Lett. 79, 10 (1997).

${ }^{32}$ V. I. Marconi, Phys. Rev. Lett. 98, 047006 (2007).

${ }^{33}$ I. Zapata, R. Bartussek, F. Sols, and P. Hänggi, Phys. Rev. Lett. 77, 2292 (1996).

${ }^{34}$ I. Zapata, J. Luczka, F. Sols, and P. Hänggi, Phys. Rev. Lett. 80, 829 (1998).

${ }^{35}$ F. Falo, P. J. Martinez, J. J. Mazo, and S. Cilla, Europhys. Lett. 45, 700 (1999).

${ }^{36}$ B. Chesca, D. John, M. Kemp, J. Brown, and C. J. Mellor, Appl. Phys. Lett. 103, 092601 (2013).

${ }^{37}$ B. Chesca, D. John, and C. J. Mellor, Supercond. Sci. Technol. 27, 055019 (2014).

${ }^{38}$ B. Chesca, D. John, and C. J. Mellor, Supercond. Sci. Technol. 27, 085015 (2014).

${ }^{39}$ J. H. Miller, G. H. Gunaratne, J. Huang, and T. D. Golding, Appl. Phys. Lett. 59, 3330-3332 (1991).

${ }^{40}$ K. K. Likharev and V. K. Semenov, IEEE Trans. Appl. Supercond. 1, 3 (1991); P. Bunyk, K. K. Likharev, and D. Zinoviev, Int. J. High Speed Electron. Syst. 11, 257 (2001).

${ }^{41}$ B. Chesca, D. John, and C. J. Mellor, Appl. Phys. Lett. 107, 162602 (2015).

${ }^{42}$ P. Caputo, J. Oppenländer, Ch. Häussler, J. Tomes, A. Friesch, T. Träuble, and N. Schopohl, Appl. Phys. Lett. 85, 1389 (2004).

${ }^{43}$ E. E. Mitchell, K. E. Hannam, J. Y. Lazar, K. E. Leslie, A. Grancea, S. T. Keenan, S. K. H. Lam, and C. P. Foley, Supercond. Sci. Technol. 29, 06LT01 (2016).

${ }^{44}$ N. F. Pedersen and A. V. Ustinov, Supercond. Sci. Technol. 8, 389 (1995). 